\title{
O TRABALHO DO ENFERMEIRO NA ORGANIZAÇÃO DOS SERVIÇOS DE SAÚDE E SUA INSERÇÃO NO DEPARTAMENTO DE INFORMÁTICA DA SMS-RP*
}

\author{
Silvana Martins Mishima** \\ Thaís Helena de Oliveira*** \\ Ione Carvalho Pinto ****
}

MISHIMA, S.M.; OLIVEIRA, T.H.de; PINTO, I.C. O trabalho do enfermeiro na organização dos serviços de saúde e sua inserção no Departamento de Informática da SMS-RP. Rev.latino-am.enfermagem, Ribeirão Preto, v. 7, n. 4, p. 13-20, outubro 1999.

Objetivamos analisar a historicidade do trabalho do enfermeiro na organização do trabalho em saúde no Departamento de Informática da Secretaria Municipal de Saúde de Ribeirão Preto, frente ao processo de municipalização. Verificamos que o enfermeiro esteve envolvido na organização desta área desde sua implantação, sendo que sua presença volta-se para a organização e controle das atividades desenvolvidas relativas à produção, armazenamento e análise dos dados necessários a diferentes etapas da assistência à saúde, bem como para aquelas voltadas ao treinamento e supervisão do pessoal que atua no sistema de informação.

UNITERMOS: enfermagem, municipalização, organização do trabalho

\section{INTRODUÇÃO}

Entendemos que os fatos históricos podem contribuir enquanto determinantes dos acontecimentos atuais e estes podem, concomitantemente, criar um processo de (re)articulação das práticas presentes na sociedade.

Neste estudo, objetivamos compreender, no processo de conformação do sistema de saúde em um dado município, a organização do trabalho do enfermeiro que atua na área básica de atenção à saúde, tomando como referência a inserção deste em uma área específica, no caso o Departamento de Informática da Secretaria Municipal de Saúde de Ribeirão Preto (SMS-RP).

A formulação do Sistema Único de Saúde-SUS, ressalta o compromisso dos municípios, de garantir aos seus cidadãos, um completo bem estar físico, psíquico e social, tomando como ponto de partida os seus princípios diretivos e organizativos (a universalidade, eqüidade, regionalização e hierarquização, participação da comunidade), na orientação da constituição do sistema no nível local.

No município de Ribeirão Preto, o processo de municipalização em saúde, trouxe para os serviços de saúde intensas transformações nos dez últimos anos. Houve uma ampliação da estrutura física da rede básica (de 13 Postos de Atenção Médica, em 1984, para 30 unidades de saúde em 1996) além da transformação do perfil quali-quantitativo dos recursos humanos.

Sendo o conjunto das mudanças no processo de trabalho em saúde articulado à finalidade do trabalho, aos instrumentos que são incorporados nos diferentes níveis de atendimento, o processo de municipalização passa a exigir a introdução de outros profissionais (físicos, psicólogos, fisioterapeutas, terapeutas ocupacionais, farmacêuticos, analistas de sistemas, digitadores, dentre outros).

Aliada a esta questão, a inserção de novas tecnologias ${ }^{1}$ neste contexto de trabalho contribui significativamente para o desenvolvimento do setor saúde, tanto no que se refere as questões de caráter assistencial quanto aquelas de caráter organizativo.

* O estudo faz parte do Projeto Integrado de Pesquisa: O trabalho de enfermagem na organização das práticas de saúde, processo CNPq n'522264/94-8

** Professora Doutora do Departamento de Enfermagem Materno-Infantil e Saúde Pública da Escola de Enfermagem de Ribeirão Preto da Universidade de São Paulo

*** Enfermeira, ex-Bolsista de Iniciação Científica da FAPESP

**** Professora Assistente do Departamento de Enfermagem Materno-Infantil e Saúde Pública da Escola de Enfermagem de Ribeirão Preto da Universidade de São Paulo

${ }^{1}$ Novas tecnologias: avanços científicos tanto no que diz respeito aos conhecimentos produzidos, como equipamentos e instrumentos técnicos 
Até 1983, a SMS-RP não dispunha, para a organização de seus serviços, nem mesmo de uma base de dados quantitativa. Segundo PEREIRA (1993), somente em 1985 deu-se o início a implantação do Sistema de Registro de Dados (boletins de produção), sendo que inúmeras dificuldades ocorriam no seu desenvolvimento, tais como: existência de processos multiplicados para quem atendia e registrava as informações nos instrumentos de coleta, baixo retorno para o nível gerador das informações, descontinuidade de processos.

No momento, o município de Ribeirão Preto conta com toda a rede básica de serviços de saúde informatizada, sendo a rede interligada através do Sistema Hygia $^{2}$. O sistema funciona ligando as unidades de saúde, laboratórios e nível central, propiciando segurança de acesso, sendo que a digitação e produção de relatórios estatísticos ficam no próprio nível local, possibilitando a constituição de um instrumento imediato na tomada de decisões para a reorganização dos serviços.

É possível evidenciar que uma "nova organização" do sistema público de saúde vem se conformando, a partir da regulamentação do Sistema Único de Saúde, e que as práticas de saúde devem se (re)articular para não só atender, mas prevenir a doença e promover a saúde. Dentro deste processo, o enfermeiro que até 1983 não fazia parte do quadro de trabalhadores da SMS-RP passa a ser incorporado à rede de serviços.

Em 1997, a rede básica de saúde contava com $154^{3}$ enfermeiros distribuídos em todas as unidades prestadoras de atenção à saúde no nível local, nas coordenações de programas de atenção à saúde (aleitamento materno, programa de assistência à saúde do escolar, serviço de assistência domiciliar, atenção fitoterápica) e nos setores específicos (vigilância epidemiológica, vigilância sanitária, planejamento, informática, etc), representando, este número de enfermeiros, um crescimento de $17.200 \%$ no período de 14 anos (1983 - 1997).

ALMEIDA et al. (1994) afirmam que, no processo de constituição e ampliação da assistência à saúde na rede básica municipal que se instala no município, principalmente com o advento das Ações Integradas de Saúde-AIS, o enfermeiro assumiu a coordenação do serviços de enfermagem e a organização das atividades meio para que o processo assistencial se processasse.

Neste sentido, temos aqui a hipótese de que o enfermeiro tem sido o profissional que, no desenvolvimento das atividades organizativas na rede básica de saúde, tem estado mais próximo da implantação e consolidação das estruturas que sustentam a conformação do sistema de informação no processo de municipalização em Ribeirão Preto.

Assim, o objetivo deste estudo é analisar a historicidade do trabalho do enfermeiro na organização do trabalho em saúde, em uma área específica de atuação - o Departamento de Informática da SMS-RP, face ao processo de municipalização.

\section{O PERCURSO DA PESQUISA}

A investigação foi realizada no Departamento de Informática da Secretaria Municipal da Saúde de Ribeirão Preto (SMS-RP), município localizado no interior do Estado de São Paulo, que conta com uma população estimada para o ano de 1997 de 557.722 habitantes ${ }^{4}$ e, dentro do processo de municipalização na saúde, encontra-se em gestão semi-plena ${ }^{5}$, desde o final de 1994.

Ribeirão Preto conta com ampla rede de serviços de saúde (públicos, privados e filantrópicos). Essa rede de serviços é constituída no âmbito hospitalar por 11 hospitais, sendo 9 gerais (1 hospital-escola de caráter público, 3 filantrópicos e 5 privados) e 2 psiquiátricos (1 público e 1 filantrópico) e na rede básica por 33 unidades de saúde - Unidades Básicas de Saúde e Unidades Básicas e Distritais de Saúde.

A SMS-RP conta com vários departamentos e divisões de caráter administrativo como o Departamento de Informática, Departamento de Vigilância Epidemiológica, Departamento de Planejamento,

\footnotetext{
${ }^{2}$ O Sistema Hygia é um software desenvolvido para a área da saúde, que tem como característica básica ser um banco de dados único, com cadastro único de paciente, agendamento de consultas com hora marcada, informatização da área laboratorial com acesso on line, registro individual e produção da área de imunização, prontuário eletrônico da clientela, elaboração de registros estatísticos de produção e faturamento

${ }^{3}$ Dados de Junho de 1997, segundo os dados do Departamento de Enfermagem da Secretaria da Municipal da Saúde de Ribeirão Preto

${ }^{4}$ Segundo o Departamento de Planejamento em Saúde da SMS-RP - dados preliminares do Censo de 1996 - IBGE

5 "A Secretaria Municipal de Saúde assume a completa responsabilidade sobre a gestão da prestação de serviços: planejamento, cadastramento, contratação, controle e pagamento de prestadores ambulatoriais e hospitalares públicos e privado; assume o gerenciamento de toda a rede pública existente no município exceto unidades hospitalares de referência sob gestão estadual; assume a execução e controle das ações básicas de saúde, nutrição e educação, de vigilância epidemiológica, de vigilância sanitária e de saúde do trabalhador no seu território, conforme definido na Comissão Bipartite, recebe mensalmente o total dos recursos financeiros para custeio correspondentes aos tetos ambulatorial e hospitalar estabelecidos" (BRASIL, 1993, p. 6963)
} 
Departamento de Enfermagem, Divisão Odontológica, Divisão Médica, Divisão de Farmácia, dentre outros.

Para a coleta de dados foi aplicado um formulário visando obter informações necessárias (quantitativo de trabalhadores, formação, função desempenhada, ano de ingresso e de desligamento) sobre os recursos humanos existentes na instituição e, mais especificamente, no Departamento de Informática. $\mathrm{O}$ formulário foi aplicado no próprio Departamento de Informática da SMS-RP respondido por dois enfermeiros que faziam parte do quadro de funcionários do mesmo.

Ainda, foi realizado o levantamento de fontes documentais, selecionadas a partir da dissertação de mestrado de PEREIRA (1993), relacionadas com a implantação do Departamento de Informática e as transformações na política de saúde do município. Estes e outros documentos foram identificados e localizados no próprio Departamento de Informática, no Departamento de Planejamento da SMS-RP e no Arquivo Público e Histórico de Ribeirão Preto - Casa da Memória. Tais fontes documentais (consultas a arquivos, fichários, portarias e normas, relativas ao desenvolvimento do trabalho do Departamento), foram confrontadas com os dados quantitativos possibilitando evidenciar os momentos das transformações na política de saúde de Ribeirão Preto e a inserção do profissional enfermeiro neste espaço.

\section{O ENFERMEIRO E SUA INSERÇÃO NO DEPARTAMENTO DE INFORMÁTICA}

Os profissionais presentes no Departamento de Informática somavam, em 1996, 28 trabalhadores, devendo-se considerar que um deles era estagiário da CODERP (Companhia de Desenvolvimento de Ribeirão Preto), e portanto, não pertencente ao quadro formal de trabalhadores da SMS-RP.

Tabela 1 - Distribuição numérica e percentual de trabalhadores do Departamento de Informática SMSRP por categoria profissional no ano de 1996. Ribeirão Preto, 1996

\begin{tabular}{l|c|c}
\hline \multicolumn{1}{c|}{ Categoria profissional } & Número & \% \\
\hline Médico & 1 & 3,57 \\
\hline Enfermeiro & 6 & 21,42 \\
\hline Analista de Sistema & 1 & 3,57 \\
\hline Digitador & 3 & 10,72 \\
\hline Técnicos de Processamento & 7 & 25,01 \\
\hline Oficial Administrativo & 3 & 10,72 \\
\hline Telefonista & 6 & 21,42 \\
\hline Estagiário CODERP & 1 & 3,57 \\
\hline TOTAL & 28 & 100 \\
\hline
\end{tabular}

Na Tabela 1 apresenta-se a distribuição numérica e o percentual dos trabalhadores do Departamento. Notase no quadro de profissionais atuantes, a presença de formações diversas, sendo que o enfermeiro constitui-se em um percentual significativo em relação aos outros trabalhadores. Os técnicos em processamento de dados correspondem a $25,01 \%$, sendo seguidos pelos enfermeiros e pelas telefonistas que constituem, cada categoria, a 21,42\% do total de trabalhadores.

É preciso assinalar que algumas destas categorias profissionais, como as telefonistas, os analistas de sistema e os técnicos de processamento de dados, foram inseridas muito recentemente no trabalho desenvolvido nesta área, e que os escriturários foram substituídos por oficiais administrativos. Mais adiante, estas questões serão melhor analisadas à luz das fontes documentais.

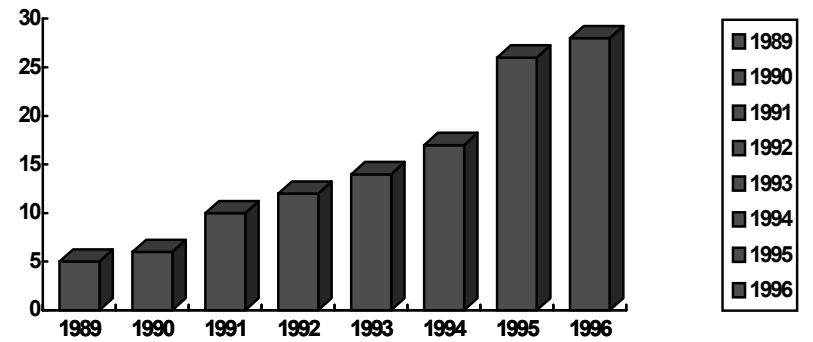

Figura 1 - Distribuição numérica do conjunto de trabalhadores do Departamento de Informática da SMS-RP para o período de 1989 à 1996. Ribeirão Preto, 1996

Pela Figura 1 é possível observar o crescimento numérico dos profissionais inseridos no Departamento de Informática de 1989 até 1996. Há um crescimento contínuo dos trabalhadores no Departamento que, no período de 1989 à 1996, representa um aumento de 460\%.

É preciso ressaltar que esse número sofre oscilações durante o período 89-96, principalmente, em função de novas contratações, sendo que as demissões são em pequeno número (apenas 1 demissão durante este período) e, ainda, da mudança de função desempenhada pelos trabalhadores de nível médio (por exemplo: funcionário "X" é admitido como escriturário em 1991 e em 1995 passa a ser técnico em processamento de dados).

Tomando como ponto de partida o ano de 1988, o ano que antecede a constituição do Núcleo de Informática (atual Departamento de Informática), procura-se analisar outros dados (fontes documentais) juntamente com os dados quantitativos, de modo a buscar uma aproximação às determinações que se fazem presentes nas etapas de desenvolvimento da implantação do Departamento de Informática.

Assim, pelo "Relatório da Área de Informações e Informática em Saúde - ano 1988" (RIBEIRÃO PRETO, 1988) constatou-se que as atividades eram desenvolvidas junto à antiga Divisão de Medicina 
Preventiva da SMS-RP, sem muita sistematização em um núcleo ou departamento específico, que trabalhasse o sistema de informações do município de Ribeirão Preto. Há indicação dos trabalhos específicos que foram desenvolvidos no decorrer deste ano, enquanto uma incipiente tentativa de estruturar os dados da área de saúde.

As atividades realizadas estavam voltadas para: processamento de boletins de atividades das Unidades Básicas de Saúde ligadas à Divisão de Medicina Preventiva; a elaboração de planilhas para cálculo de gratificação salarial; elaboração de programas que possibilitassem obter dados demográficos a partir de estimativas populacionais, a fim de agrupá-los por subsetor, faixas-etárias e "grupos alvo" para o planejamento; elaboração de planilhas que identificassem necessidades assistenciais segundo as consultas médicas esperadas, número de médicos necessários, previsão de exames laboratoriais e também internações/leitos hospitalares por área de abrangência, desde que conhecida a estimativa populacional.

As atividades desenvolvidas, portanto, voltavamse para a organização dos dados, com a estruturação de um sistema de informações que possibilitasse o planejamento de ações assistenciais a serem realizadas.

Um dos pontos relevantes deste documento refere-se a organização de um Grupo de Informações, que tinha o propósito de coordenar a elaboração e posterior movimentação do sistema de informações e sua respectiva informatização.

$\mathrm{O}$ que se pode desta forma afirmar, é que as atividades relativas à constituição de um sistema de informação na SMS-RP, têm seu início já em 1988, contando neste movimento com a figura de um médico e dois outros técnicos da área administrativa da instituição.

Nos documentos é enfatizado, em outros momentos (RIBEIRÃO PRETO, 1989), a importância da implantação de um sistema de informação para a melhoria da organização e do sistema local de saúde, dentro da perspectiva de constituição de um Sistema Único de Saúde, já identificada na $8^{\text {a }}$ Conferência Nacional de Saúde. As questões que se referem ao estabelecimento de um dado modelo assistencial e sua relação com a constituição de um sistema de informações têm sido discutidas por diferentes autores (DONALÍSIO, 1993; MENDES, 1994) e já estavam colocadas como centrais na documentação analisada.

Dentre os objetivos propostos pelo Grupo de Informação observamos: a preocupação em implantar o Sistema de Informações de modo a fortalecer a organização e melhoria do sistema local de saúde; a unificação dos processos de informação em saúde; o desenvolvimento de ações para o planejamento, controle e avaliação do sistema; o desenvolvimento de estudos epidemiológicos, administrativos e outros que possibilitassem a melhoria geral do sistema; dentre outros.
(RIBEIRÃO PRETO, 1989, p.12)

Pela análise dos documentos, neste momento histórico, pode-se notar que os mesmos indicavam as raízes da constituição do Núcleo de Informática assim como suas justificativas de implantação na rede de saúde do município em questão.

As Figuras 2 e 3 revelam que os profissionais enfermeiro, médico, digitador e escriturário compunham o quadro de funcionários no ano de 1989, que corresponde ao ano de início do Departamento de Informática, então, Núcleo de Informática da SMS-RP.

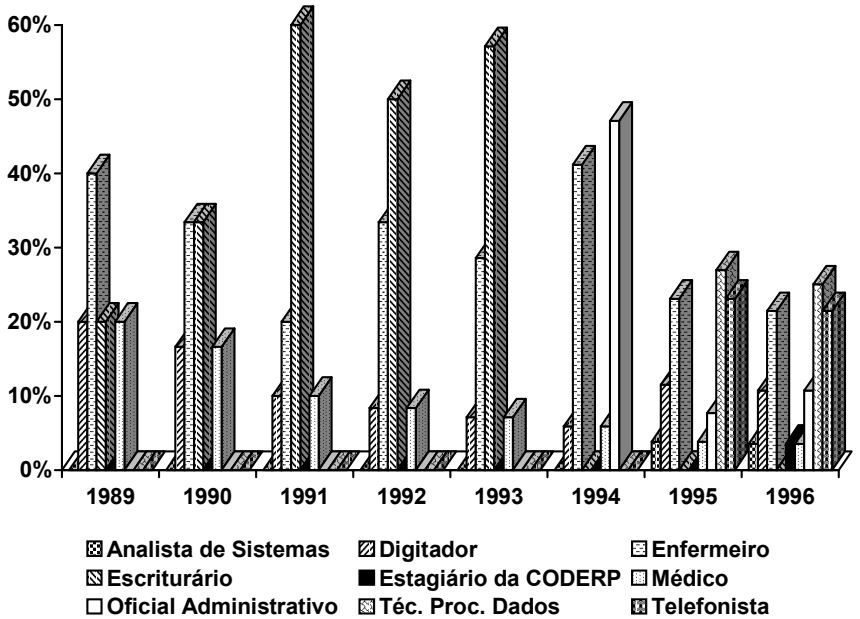

Figura 2 - Distribuição percentual dos trabalhadores do Departamento de Informática da SMS-RP por categoria profissional no período de 1989 a 1996 Ribeirão Preto - 1996

Ressalta-se ainda, que o número de enfermeiros era predominante em relação aos outros profissionais citados, somando um total de 2 (40\%), sendo 1 (20\%) médico, 1 (20\%) digitador e 1 (20\%) escriturário os outros componentes do quadro de funcionários para este ano, conforme pode ser observado pela Figura 3 .

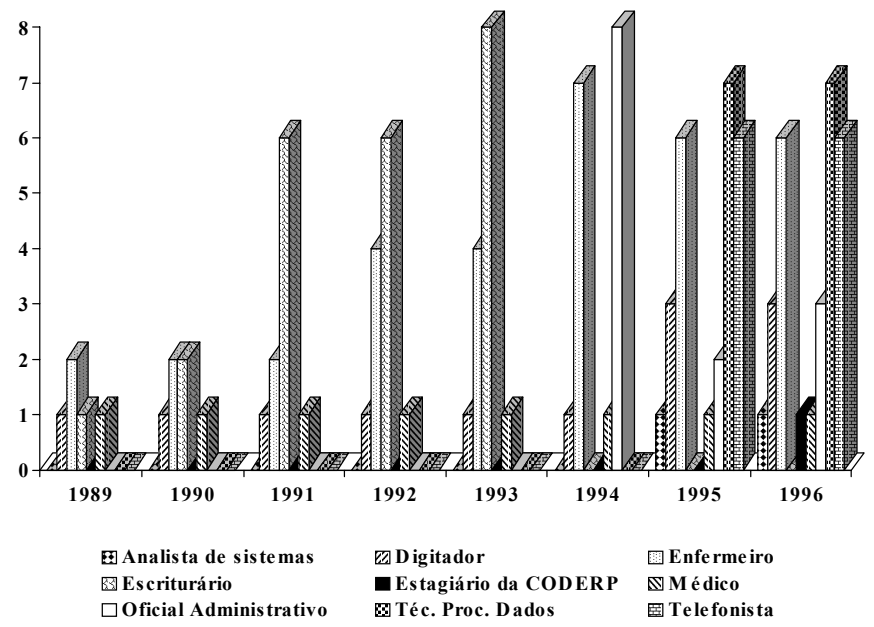

Figura 3 - Distribuição numérica de trabalhadores do Departamento de Informática da SMS-RP, por categoria profissional no período de 1989 a 1996 Ribeirão Preto - 1996 
Pode-se, portanto, observar que a partir de 1989, ao mesmo tempo em que começa a se estabelecer o Grupo de Informações e uma proposta para a estruturação de um sistema de informações no município, o enfermeiro passa a fazer parte do quadro de trabalhadores do Núcleo de Informática da SMS-RP.

Em 1990, ano do estabelecimento das leis orgânicas nos diferentes níveis de governo, o quadro de funcionários não sofre grandes alterações. Observa-se que a única categoria que sofre alteração, é a dos escriturários o que faz com que os enfermeiros e escriturários correspondam, cada grupo a 33,3\% dos trabalhadores da área de informática no ano de 1990.

As Leis Orgânicas da Saúde no 8080 e no 8142 são aprovadas em 1990, estabelece-se como uma das atribuições tanto ao nível federal, quanto estadual e municipal "a organização e coordenação do sistema de informação em saúde" (BRASIL, 1990, artigo $\mathrm{n}^{\circ} 15$, inciso IV).

A "Lei Orgânica do Município de Ribeirão Preto" publicada em abril de 1990, na Seção I - Da Saúde, observa-se na explicitação das atribuições do nível municipal, expressas no item III "planejamento das ações e implantação dos serviços de saúde com base em parâmetros populacionais" e no item $\mathrm{V}$ "instituição de sistema de referência e contra-referência com definição de território de ação das unidades de saúde" (RIBEIRÃO PRETO, 1990a) a indicação da necessidade de um sistema de informação ágil que possibilite a efetivação destas diretrizes.

Ainda em 1990, a documentação existente no município indica a constituição (RIBEIRÃO PRETO, 1990b; RIBEIRÃO PRETO, 1990c) dos recursos humanos do Grupo de Informações. Dentre os vários profissionais haviam 2 enfermeiros. Estes documentos explicitam estruturação da área de informática no âmbito da SMS-RP, tendo como pressupostos:

- "é imprescindivel que a Secretaria tenha uma estrutura direcionada às atividades de informática;

- em função do próprio organograma e tamanho/ diversidade de serviços, pressupõe uma equipe de apoio para efeito de coleta, treinamento e supervisão (grifo nosso);

- efetuar controle de qualidade nos dados coletados;

- definir prioridades e metas com uso da Informática;

- coordenar o trabalho operacional ao nível Secretaria;

- desenvolver os sistemas e aplicações onde a microinformática seja a melhor opção;

- processamento de dados em sistemas de grande porte e aplicações em micro-informática" (RIBEIRÃO PRETO, 1990b, p.4).

Segundo os documentos analisados, a opção organizacional que atendesse a estes pressupostos foi a implantação do Núcleo de Informática com a coordenação na SMS-RP integrado ao CPD-CODERP (Centro de Processamento de Dados da CODERP).

O Núcleo de Informática, então constituído, tinha como objetivos a implantação do "Sistema de Informações em Saúde" em Ribeirão Preto, de modo a fortalecer a organização e melhoria do sistema local de saúde e possibilitar uma base de dados ao "Sistema Único de Saúde" no âmbito regional, estadual e nacional (RIBEIRÃO PRETO, 1990b).

Cabe-nos ressaltar que dentre os objetivos específicos do Núcleo de Informática estavam a constituição de um banco de dados da SMS-RP, contemplando o desenvolvimento do sistema de registros estatísticos denominado "SIA-SUS - Sistema Informação Ambulatorial do Sistema Único de Saúde" para o cômputo da produção de atividades ambulatoriais para fins de financiamento do sistema, e ainda o "treinamento e supervisão relacionado ao Sistema de Informação da $S M S$-RP” (RIBEIRÃO PRETO, 1990b, p.3).

Já neste momento, observa-se a presença de um enfermeiro, pertencente à área de informática do Departamento de Medicina Preventiva da SMS-RP desde 1989, que participava da elaboração das resoluções para estruturação da área de informática no âmbito da SMSRP.

Soadas a estas questões, ainda em 1990 tem-se a publicação do "Manual de Competências da SMS-RP Resolução Interna no 33/90" (RIBEIRÃO PRETO, 1990d). Nesta resolução, formulada em outubro de 1990 não se pode identificar as competências do Núcleo de Informática, uma vez que este não se constituía da mesma forma que as estruturas formais de departamentos e divisões existentes na SMS-RP. São, entretanto, evidenciadas as competências do responsável pelo Núcleo de Informática e do enfermeiro encarregado do referido Núcleo.

Neste sentido, para o enfermeiro encarregado pelo desenvolvimento do Núcleo eram estabelecidas várias competências, dentre elas:

- "implantar o atual sistema de registro de produção nas Unidades;

- treinar e supervisionar o pessoal das Unidades para desenvolver as atividades relacionadas ao sistema de registro;

- desenvolver, implantar e avaliar o Sistema de Informações (SIA-SUDS)" (RIBEIRÃO PRETO, 1990d, p.23).

Portanto, através dos documentos há uma indicação de que o trabalho do enfermeiro voltava-se, neste momento, para as atividades relacionadas à organização e controle do processo de implantação do sistema de informação, bem como do treinamento e supervisão do pessoal que iria desenvolver atividades relacionadas ao mesmo. 
É importante assinalar, que este conjunto de aspectos em seu todo, certamente determina mudanças no perfil quantitativo e qualitativo dos trabalhadores do então Núcleo de Informática, visto que passa a ser necessário, segundo as fontes documentais, o estabelecimento de uma equipe de apoio para efeitos de coleta de dados e agregação dos mesmos, além do treinamento e supervisão dos demais trabalhadores.

Desta forma, já em 1991 tem-se um aumento significativo do número de escriturários atuantes no Núcleo (passam de 2 para 6), correspondendo a $60 \%$ dos trabalhadores, seguidos pelos enfermeiros, $20 \%$. O aumento dos escriturários parece atender às necessidades decorrentes da implantação do Sistema de Informação Ambulatorial - SIA-SUDS, e posteriormente, da "Norma Operacional $n^{\circ} 1 / 91$ " (BRASIL, 1991), editada pelo Ministério da Saúde, que passa a instituir o controle e pagamento por produção de serviços executados pelos distintos prestadores de atendimento à saúde, inclusive os municípios.

Ao nível dos municípios, a NOB 1/91 (BRASIL,1991) prevê a instituição dos Fundos de Saúde que apresentam "natureza contábil e financeira e vinculados aos objetivos do Sistema Único de Saúde [...]" (BRASIL, 1991, p.5). No município de Ribeirão Preto, a "Lei 5971 de 1991"(RIBEIRÃO PRETO, 1991b) trata do estabelecimento do Fundo Municipal de Saúde, enquanto uma instância necessária legalmente para a movimentação de recursos financeiros oriundos do Ministério da Saúde para a SMS-RP.

Embora não seja explicitamente colocado, encontra-se presente no item 7 , do artigo $4^{\circ}$ da referida Lei que "no desenvolvimento e aperfeiçoamento dos instrumentos de planejamento, gestão, administração, fiscalização e controle das ações de saúde" (RIBEIRÃO PRETO, 1991b, p.4), serão alocados recursos do Fundo Municipal de Saúde e, na forma como foi colocado neste documento, o Sistema de Informação é um instrumento necessário ao planejamento e ao processo de descentralização.

Neste sentido, os municípios, e provavelmente, o município de Ribeirão Preto, passam a ter necessidade de instituir mecanismos de registro, controle e agregação de dados de produção de serviços e, portanto, de constituir equipes que desempenhem estas atividades. $\mathrm{O}$ aumento de escriturários, principalmente em 1991, possivelmente tinha esta finalidade, ou seja, suprir a necessidade de agentes que efetivassem o registro e a agregação dos dados produzidos nas unidades locais de saúde.

Os enfermeiros presentes no Núcleo, desde 1989 continuam tendo a função de controle da produção dos registros de dados, bem como as atividades de treinamento e supervisão das equipes locais geradoras de informações. No ano de 1992, esses profissionais apresentam um crescimento de $100 \%$ em relação ao ano de 1991.

Pelo "Relatório e Estruturação de trabalho Informática SMS/RP 1991" (RIBEIRÃO PRETO, 1991a), foi possível identificar algumas necessidades do Núcleo para a sua estruturação, no que se refere à contratação de recursos humanos a fim de viabilizar o trabalho dos profissionais já atuantes. Neste sentido, algumas necessidades foram caracterizadas como urgentes, seguindo abaixo:

- "lotação do programa da CODERP em período integral para a Informática;

- alocação de um profissional com o perfil definido para a Coordenação Operacional;

- alocação de mais uma enfermeira para as tarefas relacionadas no item $I I I^{6}$ (periodo da tarde), além do trabalho na área de estatísticas vitais, área de codificação de mortalidade e morbidade;

- programação de cursos de treinamento nos softwares básicos: sistema operacional, banco de dados, planilha eletrônica e editor de textos". (RIBEIRÃO PRETO, 1991a, p.3)

Ainda em 1991, há o desenvolvimento e implantação do SIA-SUS em conjunto com a CODERP exigindo profissionais capacitados para realizar as tarefas pertinentes a este Sistema de Informação Ambulatorial do Sistema Único de Saúde. Ressaltamos aí o papel importante do enfermeiro na efetivação deste sistema em toda a rede municipal de saúde de Ribeirão Preto.

Em 1993, o total de trabalhadores do Núcleo de Informática passa a 14 , correspondendo a um crescimento de $16,6 \%$ em relação ao ano anterior, sendo o aumento em função do número de escriturários. É a partir deste ano que transformações mais significativas podem ser observadas, sendo evidenciadas através dos Gráficos 2 e 3, tais como: o aumento progressivo do número de enfermeiros e de digitadores, a transformação da função de escriturários em oficiais administrativos, que em sua maioria tiveram sua função transformada em técnico de processamento de dados em 1995, a incorporação de telefonistas e analista de sistema em 1995.

Aqui também cabe uma ressalva no sentido de indicar que estas transformações possivelmente ocorreram em função do processo de informatização que foi desencadeado no ano de 1993 na SMS-RP, com a constituição de uma rede de informações interligando os diferentes níveis do sistema de saúde, desde as Unidades Básicas de Saúde até o nível central da SMS-RP.

Em 1992 o "Projeto de implantação do sistema de agendamento informatizado da SMS-RP” (RIBEIRÃO

\footnotetext{
${ }^{6}$ As atividades referentes ao item 3 são de controle e supervisão da produção dos registros de dados
} 
PRETO, 1992) traz para o município a necessidade de:

"implantação do sistema de agendamento informatizado no sistema de saúde de Ribeirão Preto SP a curto prazo nas UBDS e ambulatórios especializados;

- sistematização do agendamento para as UBSs com retaguarda a curto prazo nas respectivas UBDS e posterior progressiva informatização" (RIBEIRÃO PRETO, 1992, p.3).

Estas preocupações são operacionalizadas na metade do ano de 1993, com o início da implantação do Sistema Hygia no município de Ribeirão Preto, já citado anteriormente.

Observando a distribuição dos profissionais atuantes no Núcleo de Informática, no ano de 1994 há um aumento significativo do número de enfermeiros (crescimento de 75\%), que são agora incorporados ao Núcleo, possivelmente na direção de contemplar a organização e o controle do novo sistema de informatização no município, bem como de atuar no treinamento e supervisão das equipes locais onde o mesmo estava sendo implantado.

É importante ressaltar que este aumento do número de enfermeiros corresponde a um crescimento real, uma vez que é possível também observar um número expressivo de oficiais administrativos (8 trabalhadores), uma categoria profissional antes inexistente. Contudo, ressalta-se que não houve a contratação destes oficiais administrativos e sim a transposição dos escriturários, que têm a função extinta na SMS-RP, para esta nova função criada a partir da "Lei complementar $n^{\circ} 361$ " (RIBEIRÃO PRETO, 1994).

Finalmente, no ano de 1995, novos trabalhadores são inseridos nesta equipe - o analista de sistemas, o técnico de processamento de dados e o telefonista, sendo este último justificado pela instituição de um sistema de agendamento de consultas por telefone, atendendo, de certa forma, às necessidades já indicadas em 1992 (RIBEIRÃO PRETO, 1992).

A presença do analista de sistema e dos técnicos em processamento de dados, se dá em função da expansão do Sistema Hygia para todo o município de Ribeirão Preto, assim como pelo aprofundamento do processo de municipalização que passa a exigir do então Departamento de Informática (a partir de 1995) maiores responsabilidades frente a produção, agregação, desagregação e utilização das informações produzidas em diferentes níveis no município.
Este quadro de transformações no perfil qualiquantitativo dos trabalhadores do Departamento de Informática a partir de 1994, ocorre certamente sofrendo influências das políticas presentes ao nível do Ministério da Saúde. Em maio de 1993, o Ministério da Saúde (BRASIL, 1993) regulamenta a Norma Operacional Básica 1/93 (NOB 1/93), onde são estabelecidas ações específicas para o aprofundamento do processo de descentralização das ações e serviços de saúde. A NOB 1/93 faz, em sua essência, cumprir as Leis n ${ }^{\circ} 8080$ e 8142 , estabelecendo formas de gestão ao nível dos municípios, enfatizando um conjunto de atividades a serem desempenhadas de acordo com o tipo de gestão assumida por cada município. Fica evidente nesta Norma, a necessidade de instituir o sistema de informação como forma de organização e gestão do sistema local de saúde.

\section{CONSIDERAÇÕES FINAIS}

O presente estudo permitiu uma aproximação à organização de uma área específica do trabalho do enfermeiro, o Departamento de Informática da SMS-RP. Este Departamento passou por inúmeras transformações acompanhando a constituição do Sistema Único de Saúde.

Pôde-se observar que o trabalho desenvolvido pelo profissional enfermeiro nesta área específica voltase, desde o início da implantação do Núcleo de Informática até sua transformação em Departamento, para a organização e controle das atividades desenvolvidas relativas à produção, armazenamento e análise dos dados necessários a diferentes etapas do processo de assistência à saúde, bem como àquelas voltadas ao treinamento e supervisão do pessoal que atua no sistema de informação ao nível local.

Foi possível identificar um crescimento contínuo do número de trabalhadores do Departamento de Informática da SMS-RP (460\% de 1989 a 1996). Atualmente, o Departamento conta com um quadro diversificado de profissionais, sendo que o enfermeiro corresponde a $21,42 \%$ dos trabalhadores, representando um número significativo desde a implantação do Departamento.

Finalmente, pode-se afirmar que as transformações quali-quantitativas dos recursos humanos do Departamento acompanharam as necessidades presentes no processo de municipalização em Ribeirão Preto.

\section{NURSES WORK IN THE ORGANIZATION OF HEALTH SERVICES AND ITS INCLUSION AT THE INFORMATICS DEPARTMENT OF THE HEALTH BUREAU OF THE CITY OF RIBEIRÃO PRETO}

The purpose of this investigation was to analyse nurse's role in the organization of the health work at the Informatics Department of the Health Bureau of the city of Ribeirão Preto, regarding the process of municipalization. We verified that nurses 
were involved in the organization of this area since its implementation. Nurses also performed organization and control activities related to the production, storage and analysis of the necessary data about the different stages of the health care, as well as training and supervising activities of the individuals who work with the information system.

KEY WORDS: nursing, municipalization, work organization

\section{EL TRABAJO DEL ENFERMERO EN LA ORGANIZACIÓN DE LOS SERVICIOS DE SALUD Y SU INSERCIÓN EN EL DEPARTAMENTO DE INFORMATICA DE LA SMS-RP}

Este trabajo tieve por objectivo analizar la historicidad del trabajo del enfermero en la organización del trabajo en salud en el Departamento de Informática de la Secretaría Municipal de Salud de Ribeirão Preto, justo el proceso de municipalización. Verificamos que el enfermero estuvo envuelto en la organización de esta área desde su implantación, siendo que su presencia se orienta hacia la organización y control de las actividades relacionadas con la producción, almacenamiento y análisis de los datos necesarios para las distintas etapas de la asistencia a la salud, así como también para aquellas orientadas hacia el entrenamiento y supervisión del personal que actua en lo sistema de información.

TÉRMINOS CLAVES: enfermería, municipalización, organización del trabajo

\section{REFERÊNCIAS BIBLIOGRÁFICAS}

01. ALMEIDA, M.C.P.; FORTUNA, C.M.; PEREIRA, M.J.B.; MISHIMA, S.M.; VILLA, T.C.S. Gerência na Rede Básica de Serviços em Ribeirão Preto - um processo em construção. Rev.Bras.Enfermagem, v. 47, n. 3, p. 278-286, 1994.

02. BRASIL. Ministério da Saúde. Lei Orgânica da Saúde $n^{\circ}$ 8080. Brasília, set. 1990.

03. BRASIL. Ministério da Saúde. Norma Técnica SUS no 1/1991. Brasília, 1991.

04. BRASIL. Ministério da Saúde. Norma Técnica SUS $\mathrm{n}^{0}$ 1/1993. Diário Oficial da União, Brasília, 20/ 05 de 1993. seção 1, p. 6961-6964.

05. DONASÍLIO, M.R. A informação e o município. Saúde Debate, n. 39, p. 64-69, 1993.

06. MENDES, E.V. Distrito sanitário: o processo social de mudança das práticas sanitárias do Sistema Único de Saúde. 2. ed. São Paulo-Rio de Janeiro: Hucitec- Abrasco, 1994.

07. PEREIRA, E.B. Municipalização da saúde em Ribeirão Preto-SP. Ribeirão Preto, 1993. 203 p. Dissertação (Mestrado) - Faculdade de Medicina de Ribeirão Preto, Universidade de São Paulo.

08. RIBEIRÃO PRETO (cidade). Secretaria Municipal de Saúde - RP. Relatório da Área de Informação e Informática em Saúde. Ribeirão Preto, dez. 1988.
09. RIBEIRÃO PRETO (cidade). Comissão Interistitucional Municipal de Saúde - RP Grupo de Informações. O trabalho de unificação de informação em saúde no município de Ribeirão Preto-SP, jan. 1989.

10. RIBEIRÃO PRETO (cidade). Prefeitura Municipal de Ribeirão Preto. Lei Orgânica da Saúde do Município de Ribeirão Preto, abril 1990a.

11. RIBEIRÃO PRETO (cidade). Secretaria Municipal de Saúde - RP. Estruturação da área de Informática no âmbito da SMS-RP e do CPDCODERP. Ribeirão Preto, agosto 1990b.

12. RIBEIRÃO PRETO (cidade). Escritório Regional de Saúde - 50. Portaria do Diretor Técnico do ERSA de 02/10/90. Ribeirão Preto, outubro 1990c.

13. RIBEIRÃO PRETO (cidade). Manual de competências - Resolução interna $\mathbf{n}^{\mathbf{0}} \mathbf{3 3} / \mathbf{9 0}$. Ribeirão Preto, outubro 1990d.

14. RIBEIRÃO PRETO (cidade). Secretaria Municipal de Saúde - RP. Relatório e Estruturação de Trabalho - Informática SMS-RP. Ribeirão Preto, março 1991a.

15. RIBEIRÃO PRETO (cidade). Lei Municipal no 5971 - Constituição do Fundo Municipal de Saúde. Ribeirão Preto, abril 1991b.

16. RIBEIRÃO PRETO (cidade). Secretaria Municipal de Saúde - RP. Projeto de Implantação do Sistema de agendamento informatizado na SMS-RP. Ribeirão Preto, 1992.

17. RIBEIRÃO PRETO (cidade). Lei complementar $\mathbf{n}^{\mathbf{0}}$ 361 de 07 de Julho de 1994. Ribeirão Preto, julho 1994. 